\title{
Existence theorems for nonlocal multivalued Hadamard fractional integro-differential boundary value problems
}

\author{
Bashir Ahmad ${ }^{1 *}$, Sotiris K Ntouyas ${ }^{1,2}$ and Ahmed Alsaedi ${ }^{1}$
}

\section{"Correspondence:}

bashir_qau@yahoo.com

${ }^{1}$ Department of Mathematics,

Faculty of Science, King Abdulaziz

University, P.O. Box 80203, Jeddah,

21589, Saudi Arabia

Full list of author information is

available at the end of the article

\begin{abstract}
In this paper, we study the existence of solutions for a boundary value problem involving Hadamard type fractional differential inclusions and integral boundary conditions. Some new existence results for convex as well as non-convex multivalued maps are obtained by using standard fixed point theorems for multivalued maps. The paper concludes with an illustrative example.

MSC: $34 \mathrm{~A} 60 ; 34 \mathrm{~A} 08$

Keywords: fractional differential inclusions; nonlocal boundary conditions; fixed point theorems
\end{abstract}

\section{Introduction}

The intensive development of fractional calculus and its widespread applications in several disciplines clearly indicate the interest of researchers and modelers in the subject. As a matter of fact, the tools of fractional calculus have been effectively used in applied and technical sciences such as physics, mechanics, chemistry, engineering, biomedical sciences, control theory, etc. It has been mainly due to the fact that fractional-order operators can exhibit the hereditary properties of many materials and processes. For a detailed account of applications and recent results on initial and boundary value problems of fractional differential equations and inclusions, we refer the reader to a series of books and papers [1-13]. However, it has been noticed that most of the work on the topic involves Riemann-Liouville and Caputo type fractional differential operators. Another class of fractional derivatives that appears side by side to Riemann-Liouville and Caputo derivatives in the literature is the fractional derivative due to Hadamard, introduced in 1892 [14]. This derivative contains logarithmic function of arbitrary exponent in the kernel of the integral in its definition. Preliminary concepts and properties of Hadamard fractional derivative and integral can be found in $[2,15-22]$.

In this paper, we study the following boundary value problem with an integral nonlocal boundary condition:

$$
\left\{\begin{array}{l}
D^{\alpha} x(t) \in F(t, x(t)), \quad 1<t<e, 1<\alpha \leq 2, \\
x(1)=0, \quad A I^{\gamma} x(\eta)+B x(e)=c, \quad 1<\eta<e,
\end{array}\right.
$$

O2014 Ahmad et al.; licensee Springer. This is an Open Access article distributed under the terms of the Creative Commons Attribution License (http://creativecommons.org/licenses/by/2.0), which permits unrestricted use, distribution, and reproduction in any medium, provided the original work is properly cited. 
where $D^{\alpha}$ is the Hadamard fractional derivative of order $\alpha, I^{\gamma}$ is the Hadamard fractional integral of order $\gamma, F:[1, e] \times \mathbb{R} \rightarrow \mathcal{P}(\mathbb{R})$ is a multivalued map, $\mathcal{P}(\mathbb{R})$ is the family of all subsets of $\mathbb{R}$ and $A, B, c$ are real constants. Further, it is assumed that $B+\left[A \Gamma(\alpha)(\log \eta)^{\gamma+\alpha-1} / \Gamma(\gamma+\alpha)\right] \neq 0$.

The present paper is motivated by a recent paper of the authors [23], where problem (1.1) was considered for a single-valued case.

We establish some existence results for the problem (1.1), when the right-hand side is convex as well as non-convex valued. The first result relies on the nonlinear alternative of Leray-Schauder type. In the second result, we shall combine the nonlinear alternative of Leray-Schauder type for single-valued maps with a selection theorem due to Bressan and Colombo for lower semi-continuous multivalued maps with nonempty closed and decomposable values, while in the third result, we shall use the fixed point theorem for contraction multivalued maps due to Covitz and Nadler. The methods used are well known, however, their exposition in the framework of problem (1.1) is new.

\section{Preliminaries}

Definition 2.1 ([2]) The Hadamard derivative of fractional order $q$ for a function $g$ : $[1, \infty) \rightarrow \mathbb{R}$ is defined as

$$
D^{q} g(t)=\frac{1}{\Gamma(n-q)}\left(t \frac{d}{d t}\right)^{n} \int_{1}^{t}\left(\log \frac{t}{s}\right)^{n-q-1} \frac{g(s)}{s} d s, \quad n-1<q<n, n=[q]+1,
$$

where $[q]$ denotes the integer part of the real number $q$ and $\log (\cdot)=\log _{e}(\cdot)$.

Definition 2.2 ([2]) The Hadamard fractional integral of order $q$ for a function $g$ is defined as

$$
I^{q} g(t)=\frac{1}{\Gamma(q)} \int_{1}^{t}\left(\log \frac{t}{s}\right)^{q-1} \frac{g(s)}{s} d s, \quad q>0,
$$

provided the integral exists.

Definition 2.3 A function $x \in A C^{2}([1, e], \mathbb{R})$ is called a solution of problem (1.1) if there exists a function $v \in L^{1}([1, e], \mathbb{R})$ with $v(t) \in F(t, x(t))$, a.e. [1,e] such that $D^{\alpha} x(t)=v(t), 1<$ $\alpha \leq 2$, a.e. $[1, e]$ and $x(1)=0, A I^{\gamma} x(\eta)+B x(e)=c, 1<\eta<e$.

Lemma 2.4 ([23]) Given $y \in C([1, e], \mathbb{R})$, the unique solution of the problem

$$
\left\{\begin{array}{l}
D^{\alpha} x(t)=y(t), \quad 1<t<e, 1<\alpha \leq 2, \\
x(1)=0, \quad A I^{\gamma} x(\eta)+B x(e)=c, \quad 1<\eta<e,
\end{array}\right.
$$

is given by

$$
x(t)=I^{\alpha} y(t)+(\log t)^{\alpha-1} \frac{c-A I^{\gamma+\alpha} y(\eta)-B I^{\alpha} y(e)}{B+\frac{A \Gamma(\alpha)}{\Gamma(\gamma+\alpha)}(\log \eta)^{\gamma+\alpha-1}} .
$$


Remark 2.5 Observe that solution (2.2) for $\alpha=2$ corresponds to the one for a boundary value problem of a Cauchy-Euler type differential equation:

$$
t^{2} \frac{d^{2} x}{d t^{2}}+t \frac{d x}{d t}=y(t)
$$

\section{Existence results}

Let us recall some basic definitions on multivalued maps [24, 25].

For a normed space $(X,\|\cdot\|)$, let $\mathcal{P}_{c l}(X)=\{Y \in \mathcal{P}(X): Y$ is closed $\}, \mathcal{P}_{b}(X)=\{Y \in$ $\mathcal{P}(X): Y$ is bounded $\}, \mathcal{P}_{c p}(X)=\{Y \in \mathcal{P}(X): Y$ is compact $\}$, and $\mathcal{P}_{c p, c}(X)=\{Y \in \mathcal{P}(X):$ $Y$ is compact and convex $\}$. A multivalued map $G: X \rightarrow \mathcal{P}(X)$ is convex (closed) valued if $G(x)$ is convex (closed) for all $x \in X$. The map $G$ is bounded on bounded sets if $G(\mathbb{B})=$ $\bigcup_{x \in \mathbb{B}} G(x)$ is bounded in $X$ for all $\mathbb{B} \in \mathcal{P}_{b}(X)$ (i.e. $\left.\sup _{x \in \mathbb{B}}\{\sup \{|y|: y \in G(x)\}\}<\infty\right)$ ). $G$ is called upper semi-continuous (u.s.c.) on $X$ if for each $x_{0} \in X$, the set $G\left(x_{0}\right)$ is a nonempty closed subset of $X$, and if for each open set $N$ of $X$ containing $G\left(x_{0}\right)$, there exists an open neighborhood $\mathcal{N}_{0}$ of $x_{0}$ such that $G\left(\mathcal{N}_{0}\right) \subseteq N$. G is said to be completely continuous if $G(\mathbb{B})$ is relatively compact for every $\mathbb{B} \in \mathcal{P}_{b}(X)$. If the multivalued map $G$ is completely continuous with nonempty compact values, then $G$ is u.s.c. if and only if $G$ has a closed graph, i.e., $x_{n} \rightarrow x_{*}, y_{n} \rightarrow y_{*}, y_{n} \in G\left(x_{n}\right)$ imply $y_{*} \in G\left(x_{*}\right)$. $G$ has a fixed point if there is $x \in X$ such that $x \in G(x)$. The fixed point set of the multivalued operator $G$ will be denoted by Fix $G$. A multivalued map $G:[1, e] \rightarrow \mathcal{P}_{c l}(\mathbb{R})$ is said to be measurable if for every $y \in \mathbb{R}$, the function

$$
t \longmapsto d(y, G(t))=\inf \{|y-z|: z \in G(t)\}
$$

is measurable.

Let $C([1, e], \mathbb{R})$ denote a Banach space of continuous functions from $[1, e]$ into $\mathbb{R}$ with the norm $\|x\|=\sup _{t \in[1, e]}|x(t)|$. Let $L^{1}([1, e], \mathbb{R})$ be the Banach space of measurable functions $x:[1, e] \rightarrow \mathbb{R}$ which are Lebesgue integrable and normed by $\|x\|_{L^{1}}=\int_{1}^{e}|x(t)| d t$.

\subsection{The Carathéodory case}

Definition 3.1 A multivalued map $F:[1, e] \times \mathbb{R} \rightarrow \mathcal{P}(\mathbb{R})$ is said to be Carathéodory if

(i) $t \longmapsto F(t, x)$ is measurable for each $x \in \mathbb{R}$;

(ii) $x \longmapsto F(t, x)$ is upper semi-continuous for almost all $t \in[1, e]$;

Further a Carathéodory function $F$ is called $L^{1}$-Carathéodory if

(iii) for each $\rho>0$, there exists $\varphi_{\rho} \in L^{1}\left([1, e], \mathbb{R}^{+}\right)$such that

$$
\|F(t, x)\|=\sup \{|v|: v \in F(t, x)\} \leq \varphi_{\rho}(t)
$$

for all $\|x\| \leq \rho$ and for a.e. $t \in[1, e]$.

For each $y \in C([1, e], \mathbb{R})$, define the set of selections of $F$ by

$$
S_{F, y}:=\left\{v \in L^{1}([1, e], \mathbb{R}): v(t) \in F(t, y(t)) \text { for a.e. } t \in[1, e]\right\} .
$$

For the forthcoming analysis, we need the following lemmas.

Lemma 3.2 (Nonlinear alternative for Kakutani maps) [26] Let E be a Banach space, $C$ a closed convex subset of $E, U$ an open subset of $C$ and $0 \in U$. Suppose that $F: \bar{U} \rightarrow \mathcal{P}_{c p, c}(C)$ 
is a upper semi-continuous compact map. Then either

(i) F has a fixed point in $\bar{U}$, or

(ii) there is $a u \in \partial U$ and $\lambda \in(0,1)$ with $u \in \lambda F(u)$.

Lemma 3.3 ([27]) Let $X$ be a Banach space. Let $F:[1, e] \times X \rightarrow \mathcal{P}_{c p, c}(X)$ be an $L^{1}-$ Carathéodory multivalued map and let $\Theta$ be a linear continuous mapping from $L^{1}([1, e], X)$ to $C([1, e], X)$. Then the operator

$$
\Theta \circ S_{F}: C([1, e], X) \rightarrow \mathcal{P}_{c p, c}(C([1, e], X)), \quad x \mapsto\left(\Theta \circ S_{F}\right)(x)=\Theta\left(S_{F, x}\right)
$$

is a closed graph operator in $C([1, e], X) \times C([1, e], X)$.

Now we are in a position to prove the existence of the solutions for the boundary value problem (1.1) when the right-hand side is convex valued.

Theorem 3.4 Assume that:

$\left(\mathrm{H}_{1}\right) \quad F:[1, e] \times \mathbb{R} \rightarrow \mathcal{P}(\mathbb{R})$ is Carathéodory and has nonempty compact and convex values;

$\left(\mathrm{H}_{2}\right)$ there exists a continuous nondecreasing function $\psi:[0, \infty) \rightarrow(0, \infty)$ and a function $p \in L^{1}\left([1, e], \mathbb{R}^{+}\right)$such that

$$
\|F(t, x)\|_{\mathcal{P}}:=\sup \{|y|: y \in F(t, x)\} \leq p(t) \psi(\|x\|) \quad \text { for each }(t, x) \in[1, e] \times \mathbb{R} ;
$$

$\left(\mathrm{H}_{3}\right)$ there exists a constant $M>0$ such that

$$
\frac{\|x\|}{\psi(\|x\|)\|p\| \omega+\frac{|c|}{|\Omega|}}>1
$$

where

$$
\begin{aligned}
& \omega=\frac{1}{\Gamma(\alpha+1)}+\frac{1}{|\Omega|}\left\{\frac{|A|(\log \eta)^{\gamma+\alpha}}{\Gamma(\gamma+\alpha+1)}+\frac{|B|}{\Gamma(\alpha+1)}\right\}, \quad \text { and } \\
& \Omega=B+\frac{A \Gamma(\alpha)}{\Gamma(\gamma+\alpha)}(\log \eta)^{\gamma+\alpha-1} .
\end{aligned}
$$

Then the boundary value problem (1.1) has at least one solution on $[1, e]$.

Proof Define the operator $\Omega_{F}: C([1, e], \mathbb{R}) \rightarrow \mathcal{P}(C([1, e], \mathbb{R}))$ by

$$
\Omega_{F}(x)=\left\{\begin{array}{l}
h \in C([1, e], \mathbb{R}): \\
h(t)=\left\{\begin{array}{l}
\frac{1}{\Gamma(\alpha)} \int_{1}^{t}\left(\log \frac{t}{s}\right)^{\alpha-1} \frac{\nu(s)}{s} d s \\
\quad+\frac{(\log t)^{\alpha-1}}{\Omega}\left\{c-\frac{A}{\Gamma(\gamma+\alpha)} \int_{1}^{\eta}\left(\log \frac{\eta}{s}\right)^{\gamma+\alpha-1} \frac{\nu(s)}{s} d s\right. \\
\left.-\frac{B}{\Gamma(\alpha)} \int_{1}^{e}\left(\log \frac{e}{s}\right)^{\alpha-1} \frac{\nu(s)}{s} d s\right\},
\end{array}\right\}
\end{array}\right.
$$

for $v \in S_{F, x}$. We will show that $\Omega_{F}$ satisfies the assumptions of the nonlinear alternative of Leray-Schauder type. The proof consists of several steps. As a first step, we show that $\Omega_{F}$ is convex for each $x \in C([1, e], \mathbb{R})$. This step is obvious since $S_{F, x}$ is convex ( $F$ has convex values), and therefore we omit the proof. 
In the second step, we show that $\Omega_{F}$ maps bounded sets (balls) into bounded sets in $C([1, e], \mathbb{R})$. For a positive number $r$, let $B_{r}=\{x \in C([1, e], \mathbb{R}):\|x\| \leq r\}$ be a bounded ball in $C([1, e], \mathbb{R})$. Then, for each $h \in \Omega_{F}(x), x \in B_{r}$, there exists $v \in S_{F, x}$ such that

$$
\begin{aligned}
h(t)= & \frac{1}{\Gamma(\alpha)} \int_{1}^{t}\left(\log \frac{t}{s}\right)^{\alpha-1} \frac{\nu(s)}{s} d s+\frac{(\log t)^{\alpha-1}}{\Omega}\left\{c-\frac{A}{\Gamma(\gamma+\alpha)} \int_{1}^{\eta}\left(\log \frac{\eta}{s}\right)^{\gamma+\alpha-1} \frac{v(s)}{s} d s\right. \\
& \left.-\frac{B}{\Gamma(\alpha)} \int_{1}^{e}\left(\log \frac{e}{s}\right)^{\alpha-1} \frac{\nu(s)}{s} d s\right\} .
\end{aligned}
$$

Then, for $t \in[1, e]$, we have

$$
\begin{aligned}
|h(t)| \leq & \frac{1}{\Gamma(\alpha)} \int_{1}^{t}\left(\log \frac{t}{s}\right)^{\alpha-1} \frac{|v(s)|}{s} d s \\
& +\frac{(\log t)^{\alpha-1}}{|\Omega|}\left[|c|+\frac{|A|}{\Gamma(\gamma+\alpha)} \int_{1}^{\eta}\left(\log \frac{\eta}{s}\right)^{\gamma+\alpha-1} \frac{|v(s)|}{s} d s\right. \\
& \left.+\frac{|B|}{\Gamma(\alpha)} \int_{1}^{e}\left(\log \frac{e}{s}\right)^{\alpha-1} \frac{|v(s)|}{s} d s\right] \\
\leq & \frac{\psi(\|x\|)\|p\|}{\Gamma(\alpha)} \int_{1}^{t}\left(\log \frac{t}{s}\right)^{\alpha-1} \frac{1}{s} d s \\
& +\frac{(\log t)^{\alpha-1}}{|\Omega|}\left[|c|+\frac{|A| \psi(\|x\|)\|p\|}{\Gamma(\gamma+\alpha)} \int_{1}^{\eta}\left(\log \frac{\eta}{s}\right)^{\gamma+\alpha-1} \frac{1}{s} d s\right. \\
& \left.+\frac{|B| \psi(\|x\|)\|p\|}{\Gamma(\alpha)} \int_{1}^{e}\left(\log \frac{e}{s}\right)^{\alpha-1} \frac{1}{s} d s\right] \\
\leq & \psi(\|x\|)\|p\|\left[\frac{1}{\Gamma(\alpha+1)}+\frac{1}{|\Omega|}\left\{\frac{A(\log \eta)^{\gamma+\alpha}}{\Gamma(\gamma+\alpha+1)}+\frac{B}{\Gamma(\alpha+1)}\right\}\right]+\frac{|c|}{|\Omega|} \\
\leq & \psi(\|x\|)\|p\| \omega+\frac{|c|}{|\Omega|} .
\end{aligned}
$$

Consequently

$$
\|h\| \leq \psi(r)\|p\| \omega+\frac{|c|}{|\Omega|} .
$$

Now we show that $\Omega_{F}$ maps bounded sets into equicontinuous sets of $C([1, e], \mathbb{R})$. Let $t_{1}, t_{2}^{\prime} \in[1, e]$ with $t_{1}<t_{2}$ and $x \in B_{r}$. For each $h \in \Omega_{F}(x)$, we obtain

$$
\begin{aligned}
\left|h\left(t_{1}\right)-h\left(t_{2}\right)\right| \leq & \frac{\psi(r)\|p\|}{\Gamma(\alpha)}\left|\int_{1}^{\tau_{1}}\left(\log \frac{\tau_{1}}{s}\right)^{\alpha-1} \frac{1}{s} d s-\int_{1}^{\tau_{2}}\left(\log \frac{\tau_{2}}{s}\right)^{\alpha-1} \frac{1}{s} d s\right| \\
& +\frac{\psi(r)\|p\|\left|\left(\log \tau_{2}\right)^{\alpha-1}-\left(\log \tau_{1}\right)^{\alpha-1}\right|}{|\Omega|} \\
& \times\left[|c|+\frac{|A|}{\Gamma(\gamma+\alpha)} \int_{1}^{\eta}\left(\log \frac{\eta}{s}\right)^{\gamma+\alpha-1} \frac{1}{s} d s+\frac{|B|}{\Gamma(\alpha)} \int_{1}^{e}\left(\log \frac{e}{s}\right)^{\alpha-1} \frac{1}{s} d s\right] \\
\leq & \frac{\psi(r)\|p\| \mid}{\Gamma(\alpha)}\left|\int_{1}^{\tau_{1}}\left[\left(\log \frac{\tau_{1}}{s}\right)^{\alpha-1}-\left(\log \frac{\tau_{2}}{s}\right)^{\alpha-1}\right] \frac{1}{s} d s\right| \\
& +\frac{\psi(r)\|p\|}{\Gamma(\alpha)}\left|\int_{\tau_{1}}^{\tau_{2}}\left(\log \frac{\tau_{2}}{s}\right)^{\alpha-1} \frac{1}{s} d s\right|
\end{aligned}
$$




$$
\begin{aligned}
& +\frac{\psi(r)\|p\|\left|\left(\log \tau_{2}\right)^{\alpha-1}-\left(\log \tau_{1}\right)^{\alpha-1}\right|}{|\Omega|} \\
& \times\left[|c|+\frac{|A|}{\Gamma(\gamma+\alpha)} \int_{1}^{\eta}\left(\log \frac{\eta}{s}\right)^{\gamma+\alpha-1} \frac{1}{s} d s+\frac{|B|}{\Gamma(\alpha)} \int_{1}^{e}\left(\log \frac{e}{s}\right)^{\alpha-1} \frac{1}{s} d s\right] .
\end{aligned}
$$

Obviously the right-hand side of the above inequality tends to zero independently of $x \in B_{r}$ as $t_{2}-t_{1} \rightarrow 0$. As $\Omega_{F}$ satisfies the above three assumptions, therefore it follows by the Ascoli-Arzelá theorem that $\Omega_{F}: C([1, e], \mathbb{R}) \rightarrow \mathcal{P}(C([1, e], \mathbb{R}))$ is completely continuous.

In our next step, we show that $\Omega_{F}$ has a closed graph. Let $x_{n} \rightarrow x_{*}, h_{n} \in \Omega_{F}\left(x_{n}\right)$ and $h_{n} \rightarrow h_{*}$. Then we need to show that $h_{*} \in \Omega_{F}\left(x_{*}\right)$. Associated with $h_{n} \in \Omega_{F}\left(x_{n}\right)$, there exists $v_{n} \in S_{F, x_{n}}$ such that for each $t \in[1, e]$,

$$
\begin{aligned}
h_{n}(t)= & \frac{1}{\Gamma(\alpha)} \int_{1}^{t}\left(\log \frac{t}{s}\right)^{\alpha-1} \frac{v_{n}(s)}{s} d s \\
& +\frac{(\log t)^{\alpha-1}}{\Omega}\left\{c-\frac{A}{\Gamma(\gamma+\alpha)} \int_{1}^{\eta}\left(\log \frac{\eta}{s}\right)^{\gamma+\alpha-1} \frac{v_{n}(s)}{s} d s\right. \\
& \left.-\frac{B}{\Gamma(\alpha)} \int_{1}^{e}\left(\log \frac{e}{s}\right)^{\alpha-1} \frac{v_{n}(s)}{s} d s\right\} .
\end{aligned}
$$

Thus it suffices to show that there exists $v_{*} \in S_{F, x_{*}}$ such that for each $t \in[1, e]$,

$$
\begin{aligned}
h_{*}(t)= & \frac{1}{\Gamma(\alpha)} \int_{1}^{t}\left(\log \frac{t}{s}\right)^{\alpha-1} \frac{v_{*}(s)}{s} d s \\
& +\frac{(\log t)^{\alpha-1}}{\Omega}\left\{c-\frac{A}{\Gamma(\gamma+\alpha)} \int_{1}^{\eta}\left(\log \frac{\eta}{s}\right)^{\gamma+\alpha-1} \frac{v_{*}(s)}{s} d s\right. \\
& \left.-\frac{B}{\Gamma(\alpha)} \int_{1}^{e}\left(\log \frac{e}{s}\right)^{\alpha-1} \frac{\nu_{*}(s)}{s} d s\right\} .
\end{aligned}
$$

Let us consider the linear operator $\Theta: L^{1}([1, e], \mathbb{R}) \rightarrow C([1, e], \mathbb{R})$ given by

$$
\begin{aligned}
f \mapsto \Theta(v)(t)= & \frac{1}{\Gamma(\alpha)} \int_{1}^{t}\left(\log \frac{t}{s}\right)^{\alpha-1} \frac{v(s)}{s} d s \\
& +\frac{(\log t)^{\alpha-1}}{\Omega}\left\{c-\frac{A}{\Gamma(\gamma+\alpha)} \int_{1}^{\eta}\left(\log \frac{\eta}{s}\right)^{\gamma+\alpha-1} \frac{v(s)}{s} d s\right. \\
& \left.-\frac{B}{\Gamma(\alpha)} \int_{1}^{e}\left(\log \frac{e}{s}\right)^{\alpha-1} \frac{\nu(s)}{s} d s\right\}
\end{aligned}
$$

Observe that

$$
\begin{aligned}
\left\|h_{n}(t)-h_{*}(t)\right\|= & \| \frac{1}{\Gamma(\alpha)} \int_{1}^{t}\left(\log \frac{t}{s}\right)^{\alpha-1} \frac{\left(v_{n}(s)-v_{*}(s)\right)}{s} d s \\
& +\frac{(\log t)^{\alpha-1}}{\Omega}\left\{c-\frac{A}{\Gamma(\gamma+\alpha)} \int_{1}^{\eta}\left(\log \frac{\eta}{s}\right)^{\gamma+\alpha-1} \frac{\left(v_{n}(s)-v_{*}(s)\right)}{s} d s\right. \\
& \left.-\frac{B}{\Gamma(\alpha)} \int_{1}^{e}\left(\log \frac{e}{s}\right)^{\alpha-1} \frac{\left(v_{n}(s)-v_{*}(s)\right)}{s} d s\right\} \| \rightarrow 0,
\end{aligned}
$$

as $n \rightarrow \infty$. 
Thus, it follows by Lemma 3.3 that $\Theta \circ S_{F}$ is a closed graph operator. Further, we have $h_{n}(t) \in \Theta\left(S_{F, x_{n}}\right)$. Since $x_{n} \rightarrow x_{*}$, therefore, we have

$$
\begin{aligned}
h_{*}(t)= & \frac{1}{\Gamma(\alpha)} \int_{1}^{t}\left(\log \frac{t}{s}\right)^{\alpha-1} \frac{\nu_{*}(s)}{s} d s \\
& +\frac{(\log t)^{\alpha-1}}{\Omega}\left\{c-\frac{A}{\Gamma(\gamma+\alpha)} \int_{1}^{\eta}\left(\log \frac{\eta}{s}\right)^{\gamma+\alpha-1} \frac{\nu_{*}(s)}{s} d s\right. \\
& \left.-\frac{B}{\Gamma(\alpha)} \int_{1}^{e}\left(\log \frac{e}{s}\right)^{\alpha-1} \frac{\nu_{*}(s)}{s} d s\right\},
\end{aligned}
$$

for some $v_{*} \in S_{F, x_{*}}$.

Finally, we show there exists an open set $U \subseteq C([1, e], \mathbb{R})$ with $x \notin \Omega_{F}(x)$ for any $\lambda \in(0,1)$ and all $x \in \partial U$. Let $\lambda \in(0,1)$ and $x \in \lambda \Omega_{F}(x)$. Then there exists $v \in L^{1}([1, e], \mathbb{R})$ with $v \in S_{F, x}$ such that, for $t \in[1, e]$, we have

$$
\begin{aligned}
x(t)= & \frac{1}{\Gamma(\alpha)} \int_{1}^{t}\left(\log \frac{t}{s}\right)^{\alpha-1} \frac{v(s)}{s} d s \\
& +\frac{(\log t)^{\alpha-1}}{\Omega}\left\{c-\frac{A}{\Gamma(\gamma+\alpha)} \int_{1}^{\eta}\left(\log \frac{\eta}{s}\right)^{\gamma+\alpha-1} \frac{\nu(s)}{s} d s\right. \\
& \left.-\frac{B}{\Gamma(\alpha)} \int_{1}^{e}\left(\log \frac{e}{s}\right)^{\alpha-1} \frac{\nu(s)}{s} d s\right\} .
\end{aligned}
$$

Using the computations of the second step above we have

$$
\|x\| \leq \psi(\|x\|)\|p\| \omega+\frac{|c|}{|\Omega|},
$$

which implies that

$$
\frac{\|x\|}{\psi(\|x\|)\|p\| \omega+\frac{|c|}{|\Omega|}} \leq 1
$$

In view of $\left(\mathrm{H}_{3}\right)$, there exists $M$ such that $\|x\| \neq M$. Let us set

$$
U=\{x \in C([1, e], \mathbb{R}):\|x\|<M\} .
$$

Note that the operator $\Omega_{F}: \bar{U} \rightarrow \mathcal{P}(C([1, e], \mathbb{R}))$ is upper semi-continuous and completely continuous. From the choice of $U$, there is no $x \in \partial U$ such that $x \in \lambda \Omega_{F}(x)$ for some $\lambda \in$ $(0,1)$. Consequently, by the nonlinear alternative of Leray-Schauder type (Lemma 3.2), we deduce that $\Omega_{F}$ has a fixed point $x \in \bar{U}$ which is a solution of the problem (1.1). This completes the proof.

\subsection{The lower semi-continuous case}

As a next result, we study the case when $F$ is not necessarily convex valued. Our strategy to deal with this problem is based on the nonlinear alternative of Leray-Schauder type together with the selection theorem of Bressan and Colombo for lower semi-continuous maps with decomposable values. 
Let $X$ be a nonempty closed subset of a Banach space $E$ and $G: X \rightarrow \mathcal{P}(E)$ be a multivalued operator with nonempty closed values. $G$ is lower semi-continuous (l.s.c.) if the set $\{y \in X: G(y) \cap B \neq \emptyset\}$ is open for any open set $B$ in $E$. Let $A$ be a subset of $[1, e] \times \mathbb{R}$. $A$ is $\mathcal{L} \otimes \mathcal{B}$ measurable if $A$ belongs to the $\sigma$-algebra generated by all sets of the form $\mathcal{J} \times \mathcal{D}$, where $\mathcal{J}$ is Lebesgue measurable in $[1, e]$ and $\mathcal{D}$ is Borel measurable in $\mathbb{R}$. A subset $\mathcal{A}$ of $L^{1}([1, e], \mathbb{R})$ is decomposable if for all $u, v \in \mathcal{A}$ and measurable $\mathcal{J} \subset[1, e]=J$, the function $u \chi_{\mathcal{J}}+v \chi_{\mathcal{J}-\mathcal{J}} \in \mathcal{A}$, where $\chi_{\mathcal{J}}$ stands for the characteristic function of $\mathcal{J}$.

Definition 3.5 Let $Y$ be a separable metric space and let $N: Y \rightarrow \mathcal{P}\left(L^{1}([1, e], \mathbb{R})\right)$ be a multivalued operator. We say $N$ has the property (BC) if $N$ is lower semi-continuous (l.s.c.) and has nonempty closed and decomposable values.

Let $F:[1, e] \times \mathbb{R} \rightarrow \mathcal{P}(\mathbb{R})$ be a multivalued map with nonempty compact values. Define a multivalued operator $\mathcal{F}: C([1, e] \times \mathbb{R}) \rightarrow \mathcal{P}\left(L^{1}([1, e], \mathbb{R})\right)$ associated with $F$ as

$$
\mathcal{F}(x)=\left\{w \in L^{1}([1, e], \mathbb{R}): w(t) \in F(t, x(t)) \text { for a.e. } t \in[1, e]\right\},
$$

which is called the Nemytskii operator associated with $F$.

Definition 3.6 Let $F:[1, e] \times \mathbb{R} \rightarrow \mathcal{P}(\mathbb{R})$ be a multivalued function with nonempty compact values. We say $F$ is of lower semi-continuous type (l.s.c. type) if its associated Nemytskii operator $\mathcal{F}$ is lower semi-continuous and has nonempty closed and decomposable values.

Lemma 3.7 ([28]) Let $Y$ be a separable metric space and let $N: Y \rightarrow \mathcal{P}\left(L^{1}([1, e], \mathbb{R})\right)$ be a multivalued operator satisfying the property $(B C)$. Then $N$ has a continuous selection, that is, there exists a continuous function (single-valued) $g: Y \rightarrow L^{1}([1, e], \mathbb{R})$ such that $g(x) \in N(x)$ for every $x \in Y$.

Theorem 3.8 Assume that $\left(\mathrm{H}_{2}\right),\left(\mathrm{H}_{3}\right)$, and the following condition holds:

$\left(\mathrm{H}_{4}\right) \quad F:[1, e] \times \mathbb{R} \rightarrow \mathcal{P}(\mathbb{R})$ is a nonempty compact-valued multivalued map such that

(a) $(t, x) \longmapsto F(t, x)$ is $\mathcal{L} \otimes \mathcal{B}$ measurable,

(b) $x \longmapsto F(t, x)$ is lower semi-continuous for each $t \in[1, e]$.

Then the boundary value problem (1.1) has at least one solution on $[1, e]$.

Proof It follows from $\left(\mathrm{H}_{2}\right)$ and $\left(\mathrm{H}_{4}\right)$ that $F$ is of l.s.c. type. Then from Lemma 3.7, there exists a continuous function $f: A C^{2}([1, e], \mathbb{R}) \rightarrow L^{1}([1, e], \mathbb{R})$ such that $f(x) \in \mathcal{F}(x)$ for all $x \in C([1, e], \mathbb{R})$.

Consider the problem

$$
\left\{\begin{array}{l}
D^{\alpha} x(t)=f(x(t)), \quad 1<t<e, 1<\alpha \leq 2, \\
x(1)=0, \quad A I^{\gamma} x(\eta)+B x(e)=c, \quad 1<\eta<e .
\end{array}\right.
$$

Observe that if $x \in A C^{2}([1, e], \mathbb{R})$ is a solution of (3.1), then $x$ is a solution to the problem (1.1). In order to transform the problem (3.1) into a fixed point problem, we define the 
operator $\bar{\Omega}_{F}$ as

$$
\begin{aligned}
\bar{\Omega}_{F} x(t)= & \frac{1}{\Gamma(\alpha)} \int_{1}^{t}\left(\log \frac{t}{s}\right)^{\alpha-1} \frac{f(x(s))}{s} d s \\
& +\frac{(\log t)^{\alpha-1}}{\Omega}\left\{c-\frac{A}{\Gamma(\gamma+\alpha)} \int_{1}^{\eta}\left(\log \frac{\eta}{s}\right)^{\gamma+\alpha-1} \frac{f(x(s))}{s} d s\right. \\
& \left.-\frac{B}{\Gamma(\alpha)} \int_{1}^{e}\left(\log \frac{e}{s}\right)^{\alpha-1} \frac{f(x(s))}{s} d s\right\} .
\end{aligned}
$$

It can easily be shown that $\bar{\Omega}_{F}$ is continuous and completely continuous. The remaining part of the proof is similar to that of Theorem 3.4. So we omit it. This completes the proof.

\subsection{The Lipschitz case}

Now we prove the existence of solutions for the problem (1.1) with a non-convex valued right-hand side by applying a fixed point theorem for a multivalued map due to Covitz and Nadler.

Let $(X, d)$ be a metric space induced from the normed space $(X ;\|\cdot\|)$. Consider $H_{d}$ : $\mathcal{P}(X) \times \mathcal{P}(X) \rightarrow \mathbb{R} \cup\{\infty\}$ given by

$$
H_{d}(A, B)=\max \left\{\sup _{a \in A} d(a, B), \sup _{b \in B} d(A, b)\right\},
$$

where $d(A, b)=\inf _{a \in A} d(a ; b)$ and $d(a, B)=\inf _{b \in B} d(a ; b)$. Then $\left(\mathcal{P}_{b, c l}(X), H_{d}\right)$ is a metric space and $\left(\mathcal{P}_{c l}(X), H_{d}\right)$ is a generalized metric space (see [29]).

Definition 3.9 A multivalued operator $N: X \rightarrow \mathcal{P}_{c l}(X)$ is called:

(a) $\gamma$-Lipschitz if and only if there exists $\gamma>0$ such that

$$
H_{d}(N(x), N(y)) \leq \gamma d(x, y) \quad \text { for each } x, y \in X
$$

(b) a contraction if and only if it is $\gamma$-Lipschitz with $\gamma<1$.

Lemma $3.10([30])$ Let $(X, d)$ be a complete metric space. If $N: X \rightarrow \mathcal{P}_{c l}(X)$ is a contraction, then Fix $N \neq \emptyset$.

Theorem 3.11 Assume that:

$\left(\mathrm{H}_{5}\right) F:[1, e] \times \mathbb{R} \rightarrow \mathcal{P}_{c p}(\mathbb{R})$ is such that $F(\cdot, x):[1, e] \rightarrow \mathcal{P}_{c p}(\mathbb{R})$ is measurable for each $x \in \mathbb{R}$.

( $\left.\mathrm{H}_{6}\right) H_{d}(F(t, x), F(t, \bar{x})) \leq m(t)|x-\bar{x}|$ for almost all $t \in[1, e]$ and $x, \bar{x} \in \mathbb{R}$ with $m \in$ $L^{1}\left([1, e], \mathbb{R}^{+}\right)$and $d(0, F(t, 0)) \leq m(t)$ for almost all $t \in[1, e]$.

Then the boundary value problem (1.1) has at least one solution on $[1, e]$ if

$$
\|m\| \omega+\frac{|c|}{|\Omega|}<1
$$


Proof Observe that the set $S_{F, x}$ is nonempty for each $x \in C([1, e], \mathbb{R})$ by the assumption $\left(\mathrm{H}_{5}\right)$, so $F$ has a measurable selection (see Theorem III.6 [31]). Now we show that the operator $\Omega_{F}$, defined in the beginning of proof of Theorem 3.4, satisfies the assumptions of Lemma 3.10. To show that $\Omega_{F}(x) \in \mathcal{P}_{c l}((C[1, e], \mathbb{R}))$ for each $x \in C([1, e], \mathbb{R})$, let $\left\{u_{n}\right\}_{n \geq 0} \in$ $\Omega_{F}(x)$ be such that $u_{n} \rightarrow u(n \rightarrow \infty)$ in $C([1, e], \mathbb{R})$. Then $u \in C([1, e], \mathbb{R})$ and there exists $v_{n} \in S_{F, x_{n}}$ such that, for each $t \in[1, e]$,

$$
\begin{aligned}
u_{n}(t)= & \frac{1}{\Gamma(\alpha)} \int_{1}^{t}\left(\log \frac{t}{s}\right)^{\alpha-1} \frac{v_{n}(s)}{s} d s \\
& +\frac{(\log t)^{\alpha-1}}{\Omega}\left\{c-\frac{A}{\Gamma(\gamma+\alpha)} \int_{1}^{\eta}\left(\log \frac{\eta}{s}\right)^{\gamma+\alpha-1} \frac{v_{n}(s)}{s} d s\right. \\
& \left.-\frac{B}{\Gamma(\alpha)} \int_{1}^{e}\left(\log \frac{e}{s}\right)^{\alpha-1} \frac{v_{n}(s)}{s} d s\right\} .
\end{aligned}
$$

As $F$ has compact values, we pass onto a subsequence (if necessary) to obtain that $v_{n}$ converges to $v$ in $L^{1}([1, e], \mathbb{R})$. Thus, $v \in S_{F, x}$ and for each $t \in[1, e]$, we have

$$
\begin{aligned}
v_{n}(t) \rightarrow v(t)= & \frac{1}{\Gamma(\alpha)} \int_{1}^{t}\left(\log \frac{t}{s}\right)^{\alpha-1} \frac{v(s)}{s} d s \\
& +\frac{(\log t)^{\alpha-1}}{\Omega}\left\{c-\frac{A}{\Gamma(\gamma+\alpha)} \int_{1}^{\eta}\left(\log \frac{\eta}{s}\right)^{\gamma+\alpha-1} \frac{v(s)}{s} d s\right. \\
& \left.-\frac{B}{\Gamma(\alpha)} \int_{1}^{e}\left(\log \frac{e}{s}\right)^{\alpha-1} \frac{\nu(s)}{s} d s\right\} .
\end{aligned}
$$

Hence, $u \in \Omega(x)$.

Next we show that there exists $\delta<1$ such that

$$
H_{d}\left(\Omega_{F}(x), \Omega_{F}(\bar{x})\right) \leq \delta\|x-\bar{x}\| \quad \text { for each } x, \bar{x} \in A C^{2}([1, e], \mathbb{R}) .
$$

Let $x, \bar{x} \in A C^{2}([1, e], \mathbb{R})$ and $h_{1} \in \Omega_{F}(x)$. Then there exists $v_{1}(t) \in F(t, x(t))$ such that, for each $t \in[1, e]$,

$$
\begin{aligned}
h_{1}(t)= & \frac{1}{\Gamma(\alpha)} \int_{1}^{t}\left(\log \frac{t}{s}\right)^{\alpha-1} \frac{\nu_{1}(s)}{s} d s \\
& +\frac{(\log t)^{\alpha-1}}{\Omega}\left\{c-\frac{A}{\Gamma(\gamma+\alpha)} \int_{1}^{\eta}\left(\log \frac{\eta}{s}\right)^{\gamma+\alpha-1} \frac{v_{1}(s)}{s} d s\right. \\
& \left.-\frac{B}{\Gamma(\alpha)} \int_{1}^{e}\left(\log \frac{e}{s}\right)^{\alpha-1} \frac{\nu_{1}(s)}{s} d s\right\} .
\end{aligned}
$$

By $\left(\mathrm{H}_{6}\right)$, we have

$$
H_{d}(F(t, x), F(t, \bar{x})) \leq m(t)|x(t)-\bar{x}(t)| .
$$

So, there exists $w \in F(t, \bar{x}(t))$ such that

$$
\left|\nu_{1}(t)-w\right| \leq m(t)|x(t)-\bar{x}(t)|, \quad t \in[1, e] .
$$


Define $U:[1, e] \rightarrow \mathcal{P}(\mathbb{R})$ by

$$
U(t)=\left\{w \in \mathbb{R}:\left|v_{1}(t)-w\right| \leq m(t)|x(t)-\bar{x}(t)|\right\} .
$$

Since the multivalued operator $U(t) \cap F(t, \bar{x}(t))$ is measurable (Proposition III.4 [31]), there exists a function $v_{2}(t)$ which is a measurable selection for $U$. So $v_{2}(t) \in F(t, \bar{x}(t))$ and for each $t \in[1, e]$, we have $\left|v_{1}(t)-v_{2}(t)\right| \leq m(t)|x(t)-\bar{x}(t)|$.

For each $t \in[1, e]$, let us define

$$
\begin{aligned}
h_{2}(t)= & \frac{1}{\Gamma(\alpha)} \int_{1}^{t}\left(\log \frac{t}{s}\right)^{\alpha-1} \frac{v_{2}(s)}{s} d s \\
& +\frac{(\log t)^{\alpha-1}}{\Omega}\left\{c-\frac{A}{\Gamma(\gamma+\alpha)} \int_{1}^{\eta}\left(\log \frac{\eta}{s}\right)^{\gamma+\alpha-1} \frac{v_{2}(s)}{s} d s\right. \\
& \left.-\frac{B}{\Gamma(\alpha)} \int_{1}^{e}\left(\log \frac{e}{s}\right)^{\alpha-1} \frac{v_{2}(s)}{s} d s\right\} .
\end{aligned}
$$

Thus,

$$
\begin{aligned}
\left|h_{1}(t)-h_{2}(t)\right|= & \mid \frac{1}{\Gamma(\alpha)} \int_{1}^{t}\left(\log \frac{t}{s}\right)^{\alpha-1} \frac{\left(v_{1}(s)-v_{2}(s)\right)}{s} d s \\
& +\frac{(\log t)^{\alpha-1}}{\Omega}\left\{c-\frac{A}{\Gamma(\gamma+\alpha)} \int_{1}^{\eta}\left(\log \frac{\eta}{s}\right)^{\gamma+\alpha-1} \frac{\left(v_{1}(s)-v_{2}(s)\right)}{s} d s\right. \\
& \left.-\frac{B}{\Gamma(\alpha)} \int_{1}^{e}\left(\log \frac{e}{s}\right)^{\alpha-1} \frac{\left(v_{1}(s)-v_{2}(s)\right)}{s} d s\right\} \mid \\
\leq & \left(\frac{\|m\|}{\Gamma(\alpha)} \int_{1}^{t}\left(\log \frac{t}{s}\right)^{\alpha-1} \frac{1}{s} d s\right. \\
& +\frac{(\log t)^{\alpha-1}}{|\Omega|}\left[|c|+\frac{|A|}{\Gamma(\gamma+\alpha)} \int_{1}^{\eta}\left(\log \frac{\eta}{s}\right)^{\gamma+\alpha-1} \frac{1}{s} d s\right. \\
& \left.\left.+\frac{|B|}{\Gamma(\alpha)} \int_{1}^{e}\left(\log \frac{e}{s}\right)^{\alpha-1} \frac{1}{s} d s\right]\right)\|x-\bar{x}\| \\
\leq & \left(\|m\| \omega+\frac{|c|}{|\Omega|}\right)\|x-\bar{x}\| .
\end{aligned}
$$

Hence,

$$
\left\|h_{1}-h_{2}\right\| \leq\left(\|m\| \omega+\frac{|c|}{|\Omega|}\right)\|x-\bar{x}\| .
$$

Analogously, interchanging the roles of $x$ and $\bar{x}$, we obtain

$$
H_{d}\left(\Omega_{F}(x), \Omega_{F}(\bar{x})\right) \leq \delta\|x-\bar{x}\| \leq\left(\|m\| \omega+\frac{|c|}{|\Omega|}\right)\|x-\bar{x}\| .
$$

Since $\Omega_{F}$ is a contraction, it follows by Lemma 3.10 that $\Omega_{F}$ has a fixed point $x$ which is a solution of (1.1). This completes the proof. 


\subsection{Example}

Example 3.12 Consider the problem

$$
\left\{\begin{array}{l}
D^{3 / 2} x(t) \in F(t, x(t)), \quad 1<t<e \\
x(1)=0, \quad I^{1 / 2} x(2)+x(e)=4
\end{array}\right.
$$

Here $\alpha=3 / 2, \gamma=1 / 2, \eta=2, A=1, B=1$, and $c=4$. With the given values, we find that

$$
\begin{aligned}
& \Omega=B+\frac{A \Gamma(\alpha)}{\Gamma(\gamma+\alpha)}(\log \eta)^{\gamma+\alpha-1} \simeq 1+\sqrt{\pi} \log \sqrt{2} \approx 2.228571, \\
& \omega=\frac{1}{\Gamma(\alpha+1)}+\frac{1}{\Omega}\left(\frac{|A|(\log \eta)^{\gamma+\alpha}}{\Gamma(\gamma+\alpha+1)}+\frac{|B|}{\Gamma(\alpha+1)}\right) \approx 1.197596 .
\end{aligned}
$$

Let $F:[1, e] \times \mathbb{R} \rightarrow \mathcal{P}(\mathbb{R})$ be a multivalued map given by

$$
x \rightarrow F(t, x)=\left[\frac{|x|^{5}}{|x|^{5}+3}+t^{3}+t^{2}+4, \frac{|x|^{3}}{|x|^{3}+1}+t+2\right] .
$$

For $f \in F$, we have

$$
|f| \leq \max \left(\frac{|x|^{5}}{|x|^{5}+3}+t^{3}+t^{2}+4, \frac{|x|^{3}}{|x|^{3}+1}+t+2\right) \leq 7, \quad x \in \mathbb{R} .
$$

Thus,

$$
\|F(t, x)\|_{\mathcal{P}}:=\sup \{|y|: y \in F(t, x)\} \leq 7=p(t) \psi(\|x\|), \quad x \in \mathbb{R},
$$

with $p(t)=1, \psi(\|x\|)=7$. In this case by the condition

$$
\frac{M}{\psi(M)\|p\| \omega+\frac{|c|}{|\Omega|}}>1
$$

we find that $M>10.178044$. Hence by Theorem 3.4 the problem (3.2) has a solution on $[1, e]$.

\section{Competing interests}

The authors declare that they have no competing interests.

Authors' contributions

Each of the authors, BA, SKN, and AA contributed to each part of this work equally and read and approved the final version of the manuscript.

\section{Author details}

1 'Department of Mathematics, Faculty of Science, King Abdulaziz University, P.O. Box 80203, Jeddah, 21589, Saudi Arabia. ${ }^{2}$ Department of Mathematics, University of loannina, loannina, 451 10, Greece.

\section{Acknowledgements}

This paper was supported by Deanship of Scientific Research (DSR), King Abdulaziz University, Jeddah, Saudi Arabia. The authors, therefore, acknowledge technical and financial support of KAU. 


\section{References}

1. Podlubny, I: Fractional Differential Equations. Academic Press, San Diego (1999)

2. Kilbas, AA, Srivastava, HM, Trujillo, Jj: Theory and Applications of Fractional Differential Equations. North-Holland Mathematics Studies, vol. 204. Elsevier, Amsterdam (2006)

3. Baleanu, D, Diethelm, K, Scalas, E, Trujillo, Jj: Fractional Calculus Models and Numerical Methods. Series on Complexity, Nonlinearity and Chaos. World Scientific, Boston (2012)

4. Agarwal, RP, Zhou, Y, He, Y: Existence of fractional neutral functional differential equations. Comput. Math. Appl. 59, 1095-1100 (2010)

5. Ahmad, B, Ntouyas, SK, Alsaedi, A: New existence results for nonlinear fractional differential equations with three-point integral boundary conditions. Adv. Differ. Equ. 2011, Article ID 107384 (2011)

6. Nyamoradi, N, Bashiri, T, Vaezpour, SM, Baleanu, D: Uniqueness and existence of positive solutions for singular fractional differential equations. Electron. J. Differ. Equ. 2014, 130 (2014)

7. O'Regan, D, Stanek, S: Fractional boundary value problems with singularities in space variables. Nonlinear Dyn. 71, 641-652 (2013)

8. Ahmad, B, Ntouyas, SK, Alsaedi, A: A study of nonlinear fractional differential equations of arbitrary order with Riemann-Liouville type multistrip boundary conditions. Math. Probl. Eng. 2013, Article ID 320415 (2013)

9. Zhang, L, Ahmad, B, Wang, G, Agarwal, RP: Nonlinear fractional integro-differential equations on unbounded domains in a Banach space. J. Comput. Appl. Math. 249, 51-56 (2013)

10. Liu, X, Jia, M, Ge, W: Multiple solutions of a $p$-Laplacian model involving a fractional derivative. Adv. Differ. Equ. 2013 Article ID 126 (2013)

11. Henderson, J, Ouahab, A: Fractional functional differential inclusions with finite delay. Nonlinear Anal. 70, 2091-2105 (2009)

12. Baleanu, D, Nazemi, SZ, Rezapour, S: The existence of solution for a k-dimensional system of multiterm fractional integrodifferential equations with antiperiodic boundary value problems. Abstr. Appl. Anal. 2014, Article ID 896871 (2014)

13. Wang, G, Liu, S, Baleanu, D, Zhang, L: A new impulsive multi-orders fractional differential equation involving multipoint fractional integral boundary conditions. Abstr. Appl. Anal. 2014, Article ID 932747 (2014)

14. Hadamard, J: Essai sur l'étude des fonctions données par leur développment de Taylor. J. Mat. Pure Appl. Ser. 8, 101-186 (1892)

15. Butzer, PL, Kilbas, AA, Trujillo, JJ: Compositions of Hadamard-type fractional integration operators and the semigroup property. J. Math. Anal. Appl. 269, 387-400 (2002)

16. Butzer, PL, Kilbas, AA, Trujillo, JJ: Fractional calculus in the Mellin setting and Hadamard-type fractional integrals. J. Math. Anal. Appl. 269, 1-27 (2002)

17. Butzer, PL, Kilbas, AA, Trujillo, JJ: Mellin transform analysis and integration by parts for Hadamard-type fractiona integrals. J. Math. Anal. Appl. 270, 1-15 (2002)

18. Kilbas, AA: Hadamard-type fractional calculus. J. Korean Math. Soc. 38, 1191-1204 (2001)

19. Kilbas, AA, Trujillo, JJ: Hadamard-type integrals as G-transforms. Integral Transforms Spec. Funct. 14, 413-427 (2003)

20. Jarad, F, Abdeljawad, T, Baleanu, D: Caputo-type modification of the Hadamard fractional derivatives. Adv. Differ. Equ. 2012, Article ID 142 (2012)

21. Ahmad, B, Ntouyas, SK: A fully Hadamard type integral boundary value problem of a coupled system of fractional differential equations. Fract. Calc. Appl. Anal. 17, 348-360 (2014)

22. Ahmad, B, Ntouyas, SK: On Hadamard fractional integro-differential boundary value problems. J. Appl. Math. Comput. (2014). doi:10.1007/s12190-014-0765-6

23. Ahmad, B, Ntouyas, SK: On nonlocal Hadamard fractional integro-differential boundary value problems. Preprint

24. Deimling, K: Multivalued Differential Equations. de Gruyter, Berlin (1992)

25. Hu, S, Papageorgiou, N: Handbook of Multivalued Analysis, Theory I. Kluwer Academic, Dordrecht (1997)

26. Granas, A, Dugundji, J: Fixed Point Theory. Springer, New York (2005)

27. Lasota, A, Opial, Z: An application of the Kakutani-Ky Fan theorem in the theory of ordinary differential equations. Bull. Acad. Pol. Sci., Sér. Sci. Math. Astron. Phys. 13, 781-786 (1965)

28. Frigon, M: Théorèmes d'existence de solutions d'inclusions différentielles. In: Granas, A, Frigon, M (eds.) Topological Methods in Differential Equations and Inclusions. NATO ASI Series C, vol. 472, pp. 51-87. Kluwer Academic, Dordrecht (1995)

29. Kisielewicz, M: Differential Inclusions and Optimal Control. Kluwer Academic, Dordrecht (1991)

30. Covitz, H, Nadler, SB Jr.: Multivalued contraction mappings in generalized metric spaces. Isr. J. Math. 8, 5-11 (1970)

31. Castaing, C, Valadier, M: Convex Analysis and Measurable Multifunctions. Lecture Notes in Mathematics, vol. 580. Springer, Berlin (1977) 